\title{
Estriol preserves synaptic transmission in the hippocampus during autoimmune demyelinating disease
}

\author{
Marina O Ziehn ${ }^{1,2}$, Andrea A Avedisian², Shannon M Dervin², Thomas J O'Dell ${ }^{3}$ and Rhonda R Voskuhl ${ }^{2}$
}

Cognitive deficits occur in over half of multiple sclerosis patients, with hippocampal-dependent learning and memory commonly impaired. Data from in vivo MRI and post-mortem studies in MS indicate that the hippocampus is targeted. However, the relationship between structural pathology and dysfunction of the hippocampus in MS remains unclear. Hippocampal neuropathology also occurs in experimental autoimmune encephalomyelitis (EAE), the most commonly used animal model of MS. Although estrogen treatment of EAE has been shown to be anti-inflammatory and neuroprotective in the spinal cord, it is unknown if estrogen treatment may prevent hippocampal pathology and dysfunction. In the current study we examined excitatory synaptic transmission during EAE and focused on pathological changes in synaptic protein complexes known to orchestrate functional synaptic transmission in the hippocampus. We then determined if estriol, a candidate hormone treatment, was capable of preventing functional changes in synaptic transmission and corresponding hippocampal synaptic pathology. Electrophysiological studies revealed altered excitatory synaptic transmission and paired-pulse facilitation (PPF) during EAE. Neuropathological experiments demonstrated that there were decreased levels of pre- and post-synaptic proteins in the hippocampus, diffuse loss of myelin staining and atrophy of the pyramidal layers of hippocampal cornu ammonis 1 (CA1). Estriol treatment prevented decreases in excitatory synaptic transmission and lessened the effect of EAE on PPF. In addition, estriol treatment prevented several neuropathological alterations that occurred in the hippocampus during EAE. Cross-modality correlations revealed that deficits in excitatory synaptic transmission were significantly correlated with reductions in trans-synaptic protein binding partners known to modulate excitatory synaptic transmission. To our knowledge, this is the first report describing a functional correlate to hippocampal neuropathology in any MS model. Furthermore, a treatment was identified that prevented both deficits in synaptic function and hippocampal neuropathology.

Laboratory Investigation (2012) 92, 1234-1245; doi:10.1038/labinvest.2012.76; published online 23 April 2012

KEYWORDS: EAE; estriol; hippocampus; MS; synaptic transmission

More than $50 \%$ of MS patients experience cognitive deficits, ${ }^{1,2}$ and hippocampal-dependent learning and memory is often impaired. ${ }^{3,4}$ Hippocampal atrophy has been shown in MS patients using in vivo MRI, ${ }^{5,6}$ and this atrophy was correlated with impaired performance on visuospatial memory testing, a hippocampal-dependent function. ${ }^{7}$ Data from postmortem studies in MS have demonstrated that hippocampal demyelination and neuropathology occur. ${ }^{8,9}$ This demyelination has been associated with changes in molecules modulating synaptic integrity, axonal transport and glutamate homeostasis. ${ }^{10}$ Although evidence from both in vivo MRI and post-mortem pathological studies indicates that hippocampal pathology occurs during MS, the relationship between such pathology and dysfunction remains unclear. Moreover, there are currently no treatments available to prevent hippocampal dysfunction during MS.

Demyelination, axonal loss and inflammation have been demonstrated within the central nervous system of mice with experimental autoimmune encephalomyelitis (EAE). Although most EAE studies have focused on spinal cord, only

\footnotetext{
Interdepartmental Program of Neuroscience, University of California, Los Angeles, Los Angeles, CA, USA; ${ }^{2}$ Multiple Sclerosis Program, Department of Neurology, University of California, Los Angeles, Los Angeles, CA, USA and ${ }^{3}$ Department of Physiology, David Geffen School of Medicine at the University of California, Los Angeles, Los Angeles, CA, USA

Correspondence: Dr RR Voskuhl, MD, Department of Neurology, University of California, Los Angeles, 635 Charles E Young Drive South, Neuroscience Research Building 1, Room 475D, Los Angeles, CA 90095, USA.
}

E-mail: rvoskuhl@ucla.edu

Received 7 January 2012; revised 14 March 2012; accepted 15 March 2012 
recently have studies investigated neurodegeneration within the brain. ${ }^{11-14}$ The functional correlates of these described pathologies in brain have not been elucidated. Specifically, little is known about the pathology that occurs in the hippocampus during EAE, and an understanding of how it may lead to dysfunction is lacking.

In MS, estriol, an estrogen elevated during pregnancy, is a therapeutic candidate because it has widespread effects on the immune system and the central nervous system. ${ }^{15}$ Studies have shown that MS patients have significantly decreased relapse rates during the third trimester of pregnancy, when estriol levels are most elevated, and that relapse rates rebound during the postpartum period coinciding with an abrupt decline in serum estriol levels. ${ }^{16}$ In non-pregnant MS patients, estriol treatment significantly reduced gadoliniumenhancing lesion number and volumes measured by MRI. ${ }^{17}$ The study further showed that estriol treatment significantly improved cognitive function as measured by the paced auditory serial addition task (PASAT) in relapsing-remitting MS patients; however, this could have been due, at least in part, to a practice effect.

Estrogen treatment can have both anti-inflammatory ${ }^{18,19}$ and neuroprotective effects during EAE. ${ }^{20,21}$ Estrogen treatment is clinically beneficial in several neurodegenerative disease models including those for Alzheimer's and Parkinson's diseases, ${ }^{22}$ and in other conditions such as traumatic brain injury and stroke. ${ }^{23,24}$ In vitro and in vivo studies have used estrogen to protect neurons against degeneration due to excitotoxicity, oxidative stress and apoptosis. ${ }^{25-27}$ Finally, estrogen treatment may affect hippocampal learning and memory, as previous studies have shown that estrogen treatment in rodents led to upregulated synaptic protein synthesis, ${ }^{28}$ increased activation of neuronal signaling cascades $^{29}$ and increased dendritic arborizations. ${ }^{30}$ Evidence demonstrates that estrogen treatment enhanced long-term potentiation, altered neuronal excitability and increased synaptic transmission in the hippocampus. ${ }^{31-33}$

In this study, we determine whether hippocampal synaptic protein complexes known to orchestrate functional synaptic transmission and the excitatory synaptic transmission itself are affected during EAE. Furthermore, we determine if estriol, a candidate hormone treatment, is capable of preventing hippocampal synaptic pathology and corresponding functional changes in synaptic transmission.

\section{MATERIALS AND METHODS}

\section{Animals and Treatment}

Gonadally intact female C57Bl/6 mice, aged 12-16 weeks, were bred in-house from animals purchased from Jackson Laboratories (Bar Harbor, ME, USA). All animals were maintained in accordance with the guidelines of the UCLA Chancellor's Animal Research Committee (ARC), and the PHS Policy on Humane Care and Use of Laboratory Animals. At 1 week before disease induction, 90-day release pellets of estriol (ESTRIOL) at $5 \mathrm{mg}$ dose, as well as placebo (PLAC) pellets that contain carrier binder alone (Innovative Research of America, Sarasota, FL, USA), were implanted into the scapular region subcutaneously.

\section{EAE Induction and Clinical Disease Scoring}

At 1 week after pellet implantation, active EAE was induced by immunizing with $200 \mu \mathrm{g}$ of myelin oligodendrocyte glycoprotein (MOG) peptide, amino acids 35-55 (Chiron Mimotopes, San Diego, CA, USA) and $300 \mu \mathrm{g}$ of Mycobacterium tuberculosis in complete Freund's adjuvant over two sites: the right draining inguinal and axillary lymph nodes. After 7 days, a booster immunization was delivered subcutaneously over the contralateral draining lymph nodes, as described. ${ }^{34}$ For MOG immunizations, mice were temporarily anesthetized with isofluorane. In addition, EAE mice received intraperitoneal injections of Bordatella pertussis toxin ( $500 \mathrm{ng} /$ mouse; Ptx) on days 0 and 2. Mice were monitored daily, and clinical disease severity was measured using the standard EAE grading scale. ${ }^{35}$ Clinical scores were averaged across all animals per day, yielding a mean clinical disease index per group.

\section{Slice Electrophysiology}

Mice were randomly selected for electrophysiological recording, daily, corresponding to EAE days 21 through 45 . Hippocampi were obtained from mice that were deeply anesthetized with halothane and then killed by cervical dislocation. The brain was removed and placed in cold $\left(4{ }^{\circ} \mathrm{C}\right)$, oxygenated $\left(95 \% \mathrm{O}_{2} / 5 \% \mathrm{CO}_{2}\right)$ artificial CSF (aCSF) containing $124 \mathrm{mM} \mathrm{NaCl}, 25 \mathrm{mM} \mathrm{Na}_{2} \mathrm{HCO}_{3}, 4.4 \mathrm{mM} \mathrm{KCl}, 1 \mathrm{mM}$ $\mathrm{NaH}_{2} \mathrm{PO}_{4}, 1.2 \mathrm{mM} \mathrm{MgSO}_{4}, 2 \mathrm{mM} \mathrm{CaCl}$ and $10 \mathrm{mM}$ glucose. The left hemisphere was collected for subsequent immunohistochemical staining (see below) and the hippocampus from the right hemisphere of each animal was then dissected free from the rest of the brain. The right hippocampus was then cut into $400 \mu \mathrm{m}$ thick slices perpendicular to its long axis and 3-4 slices from the extreme dorsal and ventral ends were discarded. The remaining slices were maintained in interface-type recording chambers perfused at a constant rate $(2-3 \mathrm{ml} / \mathrm{min})$ with a warmed $\left(30^{\circ} \mathrm{C}\right)$, oxygenated aCSF and allowed to recover for at least $2 \mathrm{~h}$ before each experiment. Low-resistance (5-10 M $\Omega$ ) glass microelectrodes filled with aCSF were placed into stratum radiatum of the hippocampal cornu ammonis 1 (CA1) region to record field excitatory postsynaptic potentials (fEPSPs). Presynaptic stimulation pulses were delivered once every $50 \mathrm{~s}$ to the Schaffer collateral/commissural fibers via a bipolar nichrome wire stimulating electrode. Basal synaptic transmission was examined by generating input/output curves where fiber volley amplitude (input) and fEPSP slopes (output) were compared across a range of presynaptic fiber stimulation intensities. ${ }^{36-38}$ To assess short-term plasticity, hippocampal slices were stimulated to trigger halfmaximal (50\%) fEPSPs. Paired-pulse facilitation (PPF) was measured by delivering pairs of presynaptic fiber stimulation pulses with varying interpulse intervals $(25,50,100,200$ and 
$275 \mathrm{~ms}$ ) and measured as the ratio of the slope of the fEPSP evoked by the second stimulation pulse relative to that produced by the first stimulation pulse. After a 20-min baseline recording, long-term potentiation (LTP) was elicited with high-frequency stimulation, where two trains of $100 \mathrm{~Hz}$, each with duration of $1 \mathrm{~s}$, were delivered with an intertrain interval of $10 \mathrm{~s}$. LTP was monitored and recorded for $60 \mathrm{~min}$ after high-frequency stimulation (HFS). Data acquisition and analysis were performed using the Experimenter's Workbench software package from DataWave Technologies (Loveland, CO, USA).

\section{Immunohistochemistry}

Left hemispheres were collected and fixed overnight in $4 \%$ paraformaldehyde, for each animal, and then transferred to a $30 \%$ sucrose/saline solution. Once tissues from all animals in the experiment had been collected, all samples were gelatinembedded, frozen and sliced thin using a cryostat at $-20^{\circ} \mathrm{C}$, as previously described. ${ }^{34}$ To determine hippocampal CA1 volume, tissue sections were uniform randomly collected and nissl stained using standard protocols. Specifically, every fifth sagittal $20 \mu \mathrm{m}$-thick tissue section was collected to sample $\sim 640 \mu \mathrm{m}$ of the left hemisphere (from Bregma lateral coordinates $0.12 \mathrm{~mm}$ to $1.08 \mathrm{~mm}$; from Paxinos and Franklin Mouse Brain Atlas ${ }^{39}$ ). For all other immunostaining conditions, three sagittal sections were used from each mouse in each condition. Tissue was first permeabilized in either $2 \%$ normal goat or normal rabbit serum (NGS or NRS) in $0.3 \%$ Triton X-100 in phosphate buffer solution for $30 \mathrm{~min}$ at room temperature. Sections were then blocked in $10-15 \%$ $\mathrm{NGS} / \mathrm{NRS}$ for $2 \mathrm{~h}$ at room temperature. To detect microglia, myelin basic protein and synaptic proteins, the following primary antibodies were used: polyclonal anti-Ibal 1:1000 (Wako Chemicals, Richmond, VA, USA); monoclonal myelin basic protein (MBP; 1:500); polyclonal anti-Synapsin-1 (Syn-1; 1:500); monoclonal postsynaptic density protein 95 (PSD-95; 1:500; Chemicon, Temecula, CA, USA); polyclonal neurexin II $\beta$ (NRXII $\beta ; 1: 500$ ); and polyclonal neuroligin 1 (NLG1; 1:1000; Santa Cruz Biotechnology, Santa Cruz, CA, USA). Fluorescent-conjugated secondary antibodies (goat anti-mouse Cy5 (1:750; Chemicon); goat anti-rabbit Cy3 IgG (1:1000; Vector Laboratories, Burlingame, CA, USA); and rabbit anti-goat Cy5 (1:750; Vector Labs) in 2\% NGS/NRS in TBS solution) were used to visualize staining. Positive and negative controls were performed for each staining to ensure antibody specificity. 4',6-diamidine-2'-pheynylindole dihydrochloride (DAPI; 1:1000; Invitrogen, Eugene, OR, USA) staining was used in all fluorescence staining conditions to identify nuclear DNA in all cell types.

\section{Microscopy and Stereological Analysis}

CA1 volume was estimated using the rigorous Cavalieri method. ${ }^{40,41} \mathrm{Iba} 1+$ cells morphologically representative of reactive microglia in CA1 were counted using unbiased stereology methods and presented as number of cells by area. ${ }^{42}$
By carefully delineating CA1 regions by anatomical landmarks, the area of myelin and PSD-95 staining within the CA1 were measured as percent area of immunoreactivity, using ImageJ (NIH; http://rsb.info/nih/gov/ij). To quantify Syn-1, NRXII $\beta$ and NLG1 immunoreactive puncta in CA1, puncta size threshold range was determined by measuring puncta size range in several tissues from each staining condition in many animals, and then averaging both upper and lower threshold limits. For Syn-1, this yielded a puncta size range from 0.816 to $3.264 \mu \mathrm{m}^{2}$. NRXII $\beta$ puncta size ranged from 0.1015 to $0.5075 \mu \mathrm{m}^{2}$, and NLG1 puncta size ranged from 0.0508 to $0.5075 \mu \mathrm{m}^{2}$. These ranges were then used to filter for puncta particle size throughout the experiment for all three of these synaptic stains. The number of puncta in each optical image was averaged across $z$-stack, brain section and animal to attain a composite average of synaptic puncta within the sampled hippocampal area in each condition.

\section{Statistical Analysis}

All quantitative measures are presented as mean \pm s.e.m. and analyzed using Student's $t$-test or one-way ANOVA. Post-hoc analyses were conducted only if ANOVA yielded $P<0.05$, with Bonferroni analysis. Pearson's linear regression analysis was used to determine correlations between electrophysiology and pathology using GraphPad Software Version 4.0 (San Diego, CA, USA). Cross-modality correlations have been made in other previously published work. ${ }^{43-45}$

\section{RESULTS}

Active EAE was induced using MOG 35-55 peptide and standard EAE clinical scores were attained, primarily reflecting the level of motor disability. There were three groups: placebo-treated healthy control females; placebo-treated females with EAE; and estriol-treated females with EAE (Figure 1a). Estriol-treated females with EAE had significantly decreased clinical disability scores compared with placebo-treated females with EAE, whereas healthy control females did not display clinical disease (Figure 1b). These findings were consistent with previous work from our lab and others, ${ }^{18,19,46}$ demonstrating that estriol treatment ameliorates motor disability in EAE.

\section{Synaptic Transmission and PPF Are Altered in the Hippocampus During EAE}

Excitatory synaptic transmission in the hippocampus during EAE has not been previously investigated. Synaptic transmission is dependent on functional and appropriately located synaptic protein families. Thus, basal synaptic transmission and PPF were assessed using in vitro hippocampal slices. To determine if EAE caused an effect in excitatory synaptic transmission, input/output (IO) relationships were compared in hippocampal slices from the right hemispheres of mice in each experimental group. The amplitude of presynaptic fiber volleys and the slope of the EPSPs evoked by different intensities of Schaffer Collateral fiber stimulation 


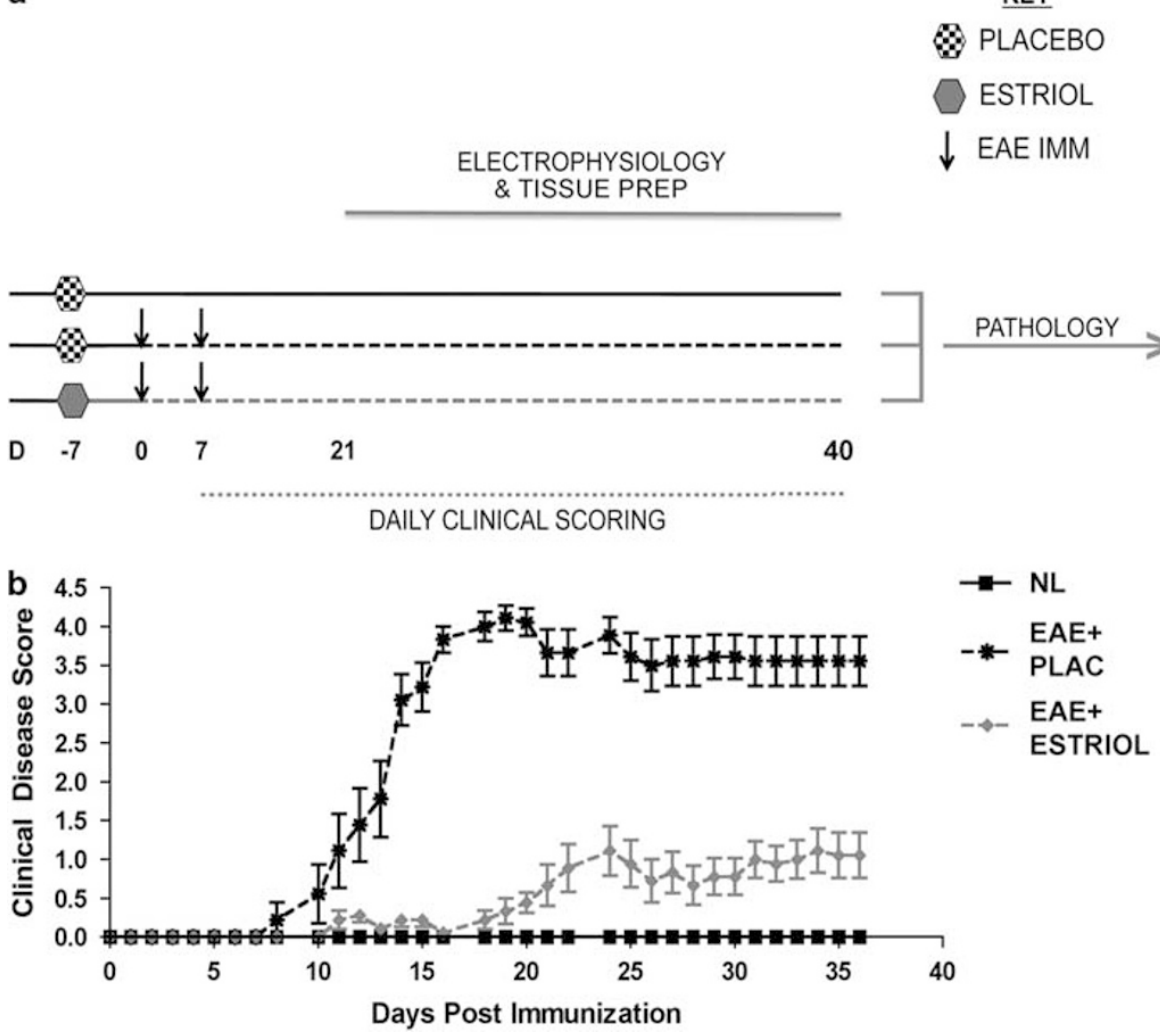

Figure 1 Experimental design and standard clinical scores in EAE. (a) Experimental design depicting the timing of placebo or estriol pellet implantation (D -7), EAE induction (D0 and D7), daily clinical scoring (D 7-40) and electrophysiology (D21-40). Pertussis injections (intraperitoneal (i.p.)) were given to both EAE groups on D0 and D2, as part of standard EAE induction (not shown). All mice were age-matched C57BI/6 adult females, and were uniform randomly selected (one per day) for electrophysiological recording. On each day of electrophysiology, the right hemisphere was prepared for electrophysiology, whereas the left hemisphere of each mouse was prepared for pathology studies and kept until all tissue from all conditions had been collected. (b) EAE clinical scores were recorded in placebo-treated (EAE + PLAC, black star, dashed black line) or estriol-treated (EAE + ESTRIOL, gray triangle, dashed gray line) mice as well as in non-EAE induced healthy controls (NL, black square, solid black line). Placebo-treated mice with EAE exhibited a severe clinical disease course, whereas estriol-treated mice exhibited significantly reduced clinical severity. Data are representative of two separate experiments, and experiment has been repeated more four times. Repeated measures ANOVA with post-hoc pair-wise comparisons revealed that placebotreated EAE mice were significantly different from other two groups beginning at D12 of disease, ${ }^{\star} P<0.05, n=5$ per group per experiment.

were analyzed. Here, hippocampal slices from placebo-treated EAE mice had a significantly altered IO curve compared with those from healthy controls (Figure 2a). Hippocampal slices from EAE mice had FV Amps and fEPSP slopes that were both decreased by nearly $50 \%$, suggesting that basal synaptic transmission was significantly impaired during EAE. When treated with estriol, however, hippocampal slices from mice with EAE displayed IO relationships more similar to healthy controls (Figure 2a and b). When maximal fEPSP slopes were compared across the three groups, there was a significant deficit in postsynaptic responses in slices from EAE mice compared with healthy controls, and this decrease in the postsynaptic response was prevented with estriol treatment (Figure 2b). Next, to examine short-term plasticity, we measured PPF at Schaffer collateral fiber inputs onto CA1 pyramidal cells. PPF is inversely correlated with the probability of neurotransmitter release from presynaptic terminals, such that an increase in PPF is indicative of a decrease in transmitter release probability, whereas a decrease in PPF is indicative of an increase in transmitter release probability. Compared with healthy controls, placebo-treated EAE mice displayed significantly increased PPF at the shortest paired-pulse interval tested (Figure $2 \mathrm{c}$ and $\mathrm{d}$ ). Interestingly, although PPF in slices from estriol-treated EAE mice was also significantly elevated compared with healthy controls, the increase tended to be lower than placebo-treated EAE mice at the same time interval (Figure $2 \mathrm{c}$ and $\mathrm{d}$ ). As hippocampal slices from placebo-treated EAE mice had significantly increased paired-pulse ratios, our results suggest that EAE caused a decrease in the probability of presynaptic neurotransmitter release. With estriol treatment during EAE there was a trend of partially restored release probability. To examine if EAE impaired synaptic plasticity, LTP was induced with HFS $(2 \times 100 \mathrm{~Hz})$ in hippocampal slices from mice in each condition. Two separate components of LTP were analyzed: post-tetanic potentiation (PTP; the immediate potentiated response of each slice after HFS stimulation) and early-phase LTP (eLTP; the average of the past $5 \mathrm{~min}$ of LTP 

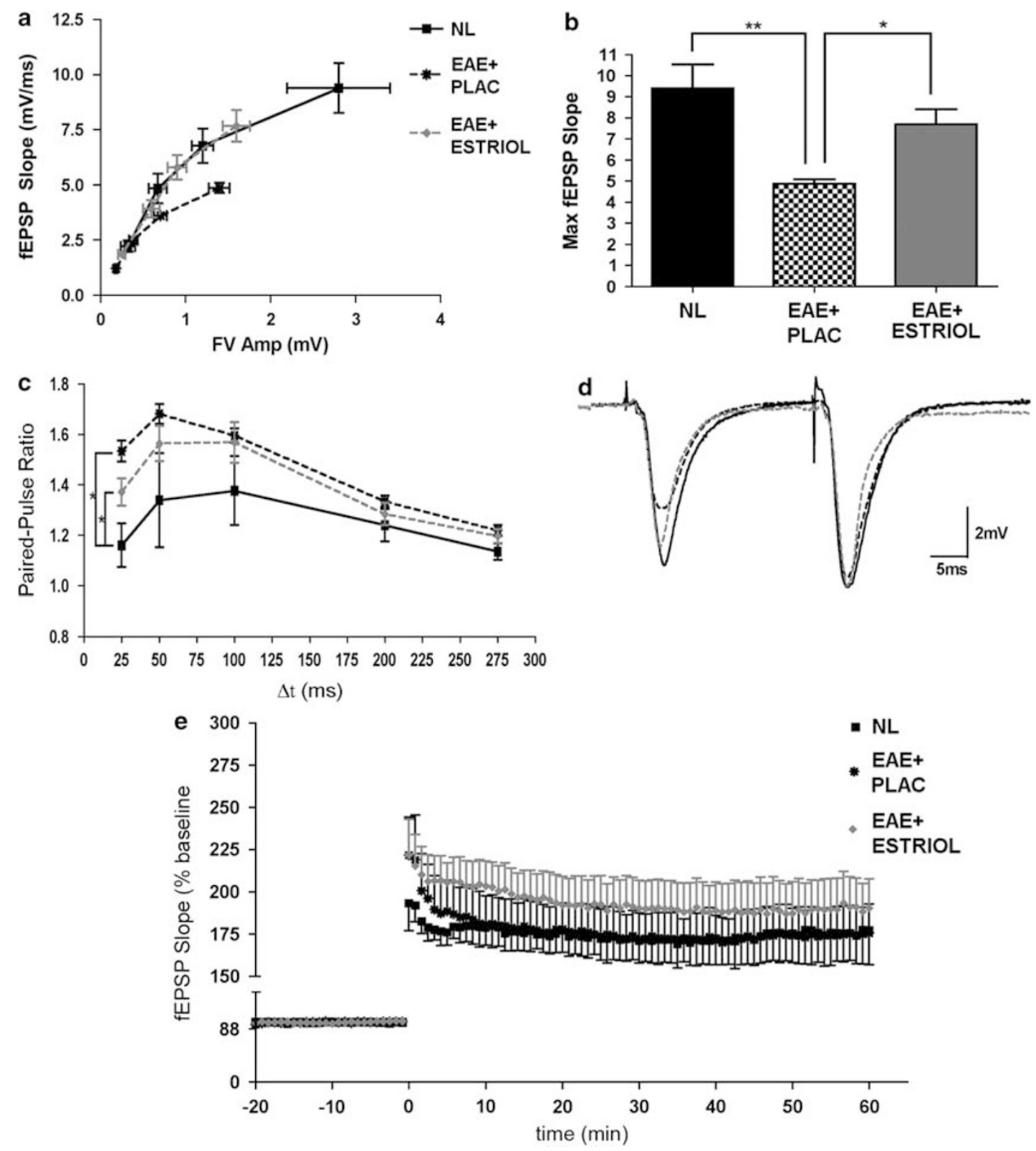

Figure 2 Excitatory synaptic transmission and paired-pulse facilitation are altered during EAE. (a) Input/output (IO) curves were created by comparing fiber volley amplitude (FV Amp; $\mathrm{mV}$ ) to field excitatory postsynaptic potential (fEPSP; $\mathrm{mV} / \mathrm{ms}$ ) during four different stimulation intensities of presynaptic fiber stimulation that caused fEPSP responses of 25, 50, 75 and 100\% maximal fEPSP amplitude. One-way ANOVA analyses demonstrated that placebo-treated EAE (EAE + PLAC; black star, dashed black line) fEPSP responses were significantly reduced at each point along the IO curve (percentage of maximal fEPSP slope: 25 , 5075 and 100\%) in comparison with healthy controls (NL; black squares, solid black line), $P<0.05$. With estriol treatment during EAE (EAE + ESTRIOL; gray triangle, dashed gray line), the IO curve followed a similar trajectory to healthy controls. (b) A histogram demonstrating decreased maximal fEPSP responses in placebo-treated EAE compared with healthy controls, and maximal fEPSPs in estriol-treated EAE mice that were not significantly different from controls. Oneway ANOVA revealed a significant effect of disease on maximal fEPSP slope $(P=0.0029)$ and follow-up Bonferroni tests indicated that placebo-treated EAE mice (EAE + PLAC; $n=15$ slices from 5 mice) were significantly different from healthy controls (NL, $n=7$ slices from 3 mice; ${ }^{* * P}<0.01$ ) and also significantly different from estriol-treated EAE (EAE + ESTRIOL, $n=16$ slices from 5 mice; ${ }^{*} P<0.05$ ). (c) Paired-pulse facilitation was increased in the hippocampal CA1 region of placebo-treated EAE mice. Paired presynaptic fiber stimulation pulses were delivered with varying interpulse intervals $(25,50,100,200$ and 275 ms) to elicit postsynaptic responses with amplitudes that were then compared. At an interpulse interval of $25 \mathrm{~ms}$, one-way ANOVA demonstrated that the condition had a significant effect on paired-pulse facilitation $(P=0.0178)$. Bonferroni post-hoc analysis showed that placebo-treated mice (EAE + PLAC; black star, dashed black line, $n=12$ slices from 5 mice) had significantly greater paired-pulse facilitation $\left({ }^{*} P<0.05\right)$ compared with healthy controls (NL; black square, solid black line, $n=6$ slices from 3 mice). Estriol-treated mice (EAE + ESTRIOL; gray triangle, dashed gray line, $n=11$ slices from 5 mice) also had significantly increased PPF compared with healthy controls. (d) Sample waveforms depict representative paired-pulse responses recorded from animals in each experimental condition at a 25-ms interpulse interval (NL: black solid line; EAE + PLAC: dashed black line; and EAE + ESTRIOL: gray dashed line). (e) Neither the post-tetanic potentiation (PTP; the first response after high-frequency stimulation) nor the early phase of LTP were significantly affected by EAE, as analyzed by one-way ANOVAs. Furthermore, one-way ANOVAs revealed that estriol treatment during EAE did not have an effect on PTP or eLTP. 

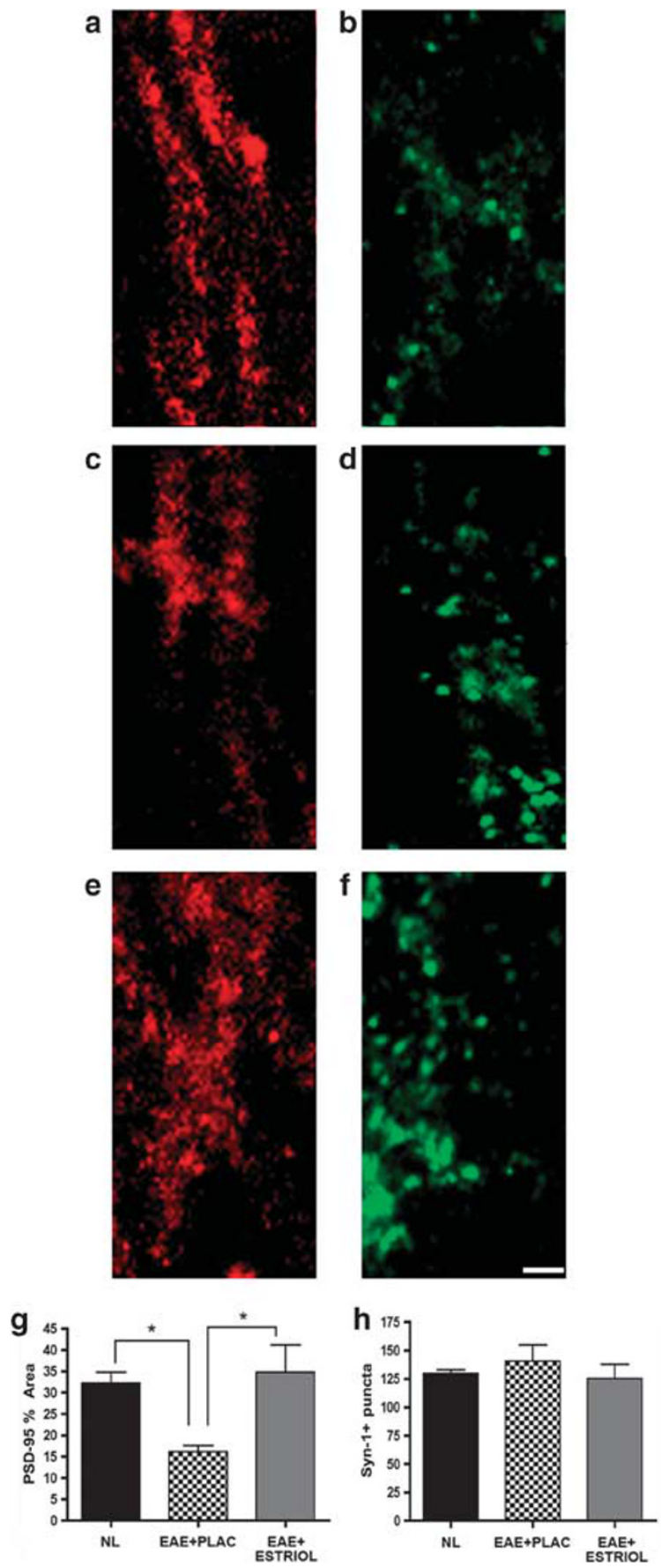

Figure 3 Hippocampal PSD-95 levels are decreased in EAE and preserved with estriol treatment. (a-f) Fluorescent images depict the stratum radiatum with the $C A 1$ region of representative hippocampal slices from healthy $(\mathbf{a}, \mathbf{b})$, placebo-treated $\operatorname{EAE}(\mathbf{c}, \mathbf{d})$ and estriol-treated $\operatorname{EAE}(\mathbf{e}, \mathbf{f})$ mice, where PSD-95 (a, c, e; Cy5-red), and Synapsin-1 (b, d, f; Syn-1; Cy3-green) staining are shown at $\times 60$ magnification. During EAE, PSD-95 percent area was significantly decreased in the CA1 region of the hippocampus (c) compared with healthy controls (a). This decrease was prevented with estriol treatment during $\operatorname{EAE}(\mathbf{e})$, which is quantified in graph g. One-way ANOVA and Bonferroni post-hoc analysis revealed statistical significance in PSD-95 percent area where ${ }^{\star} P=0.0324, n=5$ per group. Average number of presynaptic Syn-1 + puncta within the CA1 was not significantly different across conditions (h). These experiments were repeated twice with similar results. Scale bar, $1 \mu \mathrm{m}$. in each slice). When compared across groups, PTP was not significantly affected by disease or by estriol treatment during disease. eLTP was also unaffected by EAE or estriol treatment during EAE (Figure 2e).

\section{Pre- and Post-synaptic Proteins Are Differentially Affected in the Hippocampus During EAE and Estriol Treatment of EAE}

Synaptic health and transmission are critical to hippocampal functions including learning and memory. Because excitatory synaptic transmission and PPF were both affected during EAE, we next assessed whether synaptic proteins were also affected in the hippocampus during EAE. Presynaptic (Syn-1 and NRXII $\beta$ ) and postsynaptic (PSD-95 and NLG1) protein immunoreactivity was measured in the hippocampus of normal, placebo-treated EAE and estriol-treated EAE mice. The percent area of PSD-95 staining was significantly decreased in EAE mice compared with healthy controls (Figure 3a $v s \mathrm{c}$, and graph g). Estriol treatment during EAE reversed this effect on PSD-95 (Figure $3 e$ and graph g). In contrast, the number of Syn-1 puncta was not significantly reduced during EAE (Figure $3 b, d, f$ and graph $h$ ).

Recent evidence has demonstrated that PSD-95 can bind to NLG1 to modulate presynaptic release probability in a retrograde trans-synaptic fashion by binding to $\beta$-neurexin II. ${ }^{47}$ As we found that PSD-95 levels were significantly decreased in the hippocampus during EAE, we next investigated whether EAE affected levels of presynaptic NRXII $\beta$ and postsynaptic NLG1. We found that immunoreactive NRXII $\beta$ puncta were significantly decreased in hippocampi from placebo-treated EAE compared with healthy controls (Figure $4 \mathrm{a}$ and $\mathrm{b}$ ). Estriol treatment of EAE mice prevented this decrease in NRXII $\beta$ and resulted in a similar amount of immunoreactive puncta within hippocampus as that found in healthy control mice. Immunoreactive NLG1 puncta were also significantly decreased in placebotreated EAE compared with healthy controls, and estriol treatment of EAE also prevented this decrease in NLG1 (Figure $4 \mathrm{c}$ and $\mathrm{d}$ ).

\section{Demyelination and Microglial Activation Occur in the Hippocampus During EAE}

Demyelination is a classic neuropathological finding in spinal cords of mice with EAE and often leads to deficits in sensory and motor function. Demyelination has been observed in the hippocampus of MS patients ${ }^{10}$ generally as focal demyelinating lesions. We have demonstrated in a previous report that there is a diffuse reduction in myelin staining in the hippocampus during EAE. ${ }^{34}$ Thus, myelin staining was assessed in this study. MBP fluorescence intensity measurements demonstrated a significant reduction in myelin immunoreactivity within the delineated CAl region of placebo-treated EAE mice, compared with healthy controls (Figure 5a, b and d). Estriol treatment during EAE significantly preserved myelin staining in the CA1 region 

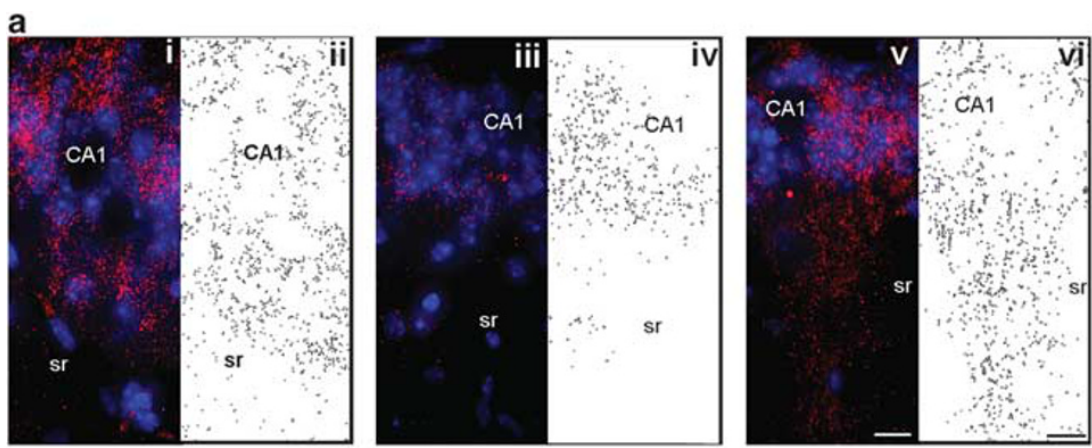

C
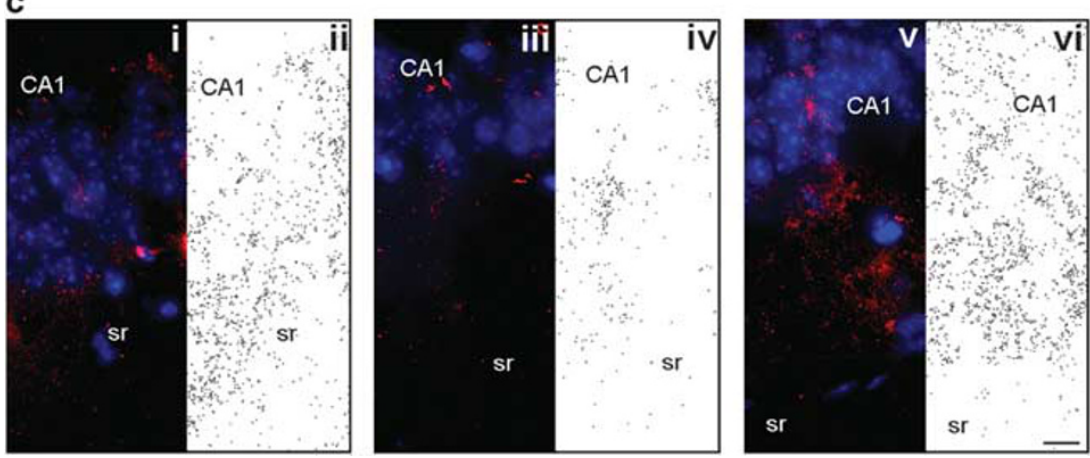
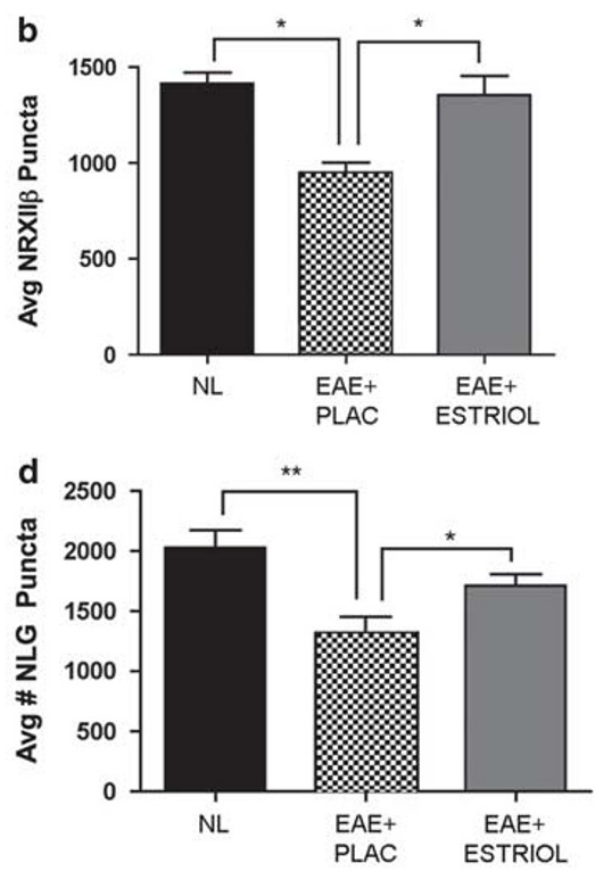

Figure 4 Presynaptic $\beta$-neurexin II (NRXII $\beta$ ) and postsynaptic neuroligin-1 (NLG1) are decreased during EAE, but preserved with estriol treatment. Synaptic puncta were measured in the hippocampal CA1 region by counting immunoreactive NRXII $\beta$ puncta $(\mathbf{a}, \mathbf{b})$ and NLG1 puncta (c, d) in healthy control (panels i, ii), placebo-treated EAE (panels iii, iv) and estriol-treated EAE mice (panels v, vi), respectively. Pseudo-colored confocal images depict synaptic staining (either NRXII $\beta$ or NLG1, Cy3-red) in hippocampus at $\times 60$ magnification, whereas black and white images show outlined puncta from respective confocal images. (b) One-way ANOVA revealed that groups differed significantly in quantity of NRXII $\beta$ puncta $(P=0.005)$, and Bonferroni post-hoc test indicated that placebo-treated EAE mice (EAE + PLAC, $n=5$ mice) had significantly decreased $(* P<0.01)$ numbers of NRXII $\beta$ puncta compared with healthy control (NL, $n=5$ mice) and estriol-treated EAE mice (EAE + ESTRIOL, $n=4$ mice). (d) Simultaneously, NLG1 puncta quantities were also significantly different across conditions (one-way ANOVA, $P=0.0056$ ) with follow-up tests indicating significant difference between healthy controls (NL, $n=5$ mice) and placebo-treated EAE mice (EAE + PLAC, ${ }^{*} P<0.01, n=5$ mice), as well as significant difference between placebo-treated EAE and estriol-treated EAE mice (EAE + ESTRIOL, ${ }^{*} P<0.05, n=5$ mice). These experiments were repeated twice and similar results were found. Scale bars, $10 \mu \mathrm{m}$.

(Figure $5 \mathrm{c}$ and $\mathrm{d}$ ) compared with placebo-treated EAE mice, but myelin staining levels were not similar to normal controls.

Reactive microglia with infiltration of $\mathrm{T}$ lymphocytes and macrophages characterize the inflammation that occurs in spinal cords of EAE mice. Within the hippocampus, however, we have previously demonstrated that immune cell infiltrates are minimal, with inflammation limited to resident reactive microglia. ${ }^{34}$ Reactive microglia and the inflammatory molecules they produce have also been shown to detrimentally affect synaptic transmission. ${ }^{14}$ Iba1 is a protein highly expressed by resident microglia in the hippocampus. ${ }^{48}$ Ibal is also expressed by macrophages, and the two cell types may be distinguished by morphology, as we have previously described, ${ }^{49}$ Thus, Ibal staining was used to label, count and morphologically characterize cells of microglial/macrophage lineage. Hippocampal tissue sections from placebo-treated EAE mice demonstrated a trend for elevated numbers of highly branched, ramified microglia compared with healthy controls, which had small, finely branched quiescent microglia (Figure $5 \mathrm{a}, \mathrm{b}$ and graph e). Estriol treatment in EAE mice tended to prevent this increase in ramified microglia (Figure $5 \mathrm{c}$ and graph e). Within the hippocampus, we did not find large, globoid Iba1-positive cells, suggesting that inflammation in the hippocampus consists primarily of reactive microglia and not immune cell infiltrates.

\section{CA1 Pyramidal Layer Volume Is Decreased During Disease and Preserved with Estriol Treatment}

The hippocampal CA1 region undergoes atrophy in humans with MS. ${ }^{7}$ As such, we next determined if atrophy was occurring in the hippocampal CA1 during, where altered synaptic transmission had also been found. CA1 volume was rigorously assessed using the Cavalieri method of volume estimation for histopathological data. EAE caused a significant decrease in CA1 pyramidal layer (CA1 pyr) volume compared with healthy controls (Figure $6 \mathrm{a}, \mathrm{b}$ and $\mathrm{d}$ ). Importantly, estriol treatment during EAE prevented CA1 pyramidal layer volume loss and preserved CA1 layer volume to levels similar to healthy controls (Figure $6 \mathrm{c}$ and $\mathrm{d}$ ). 
a

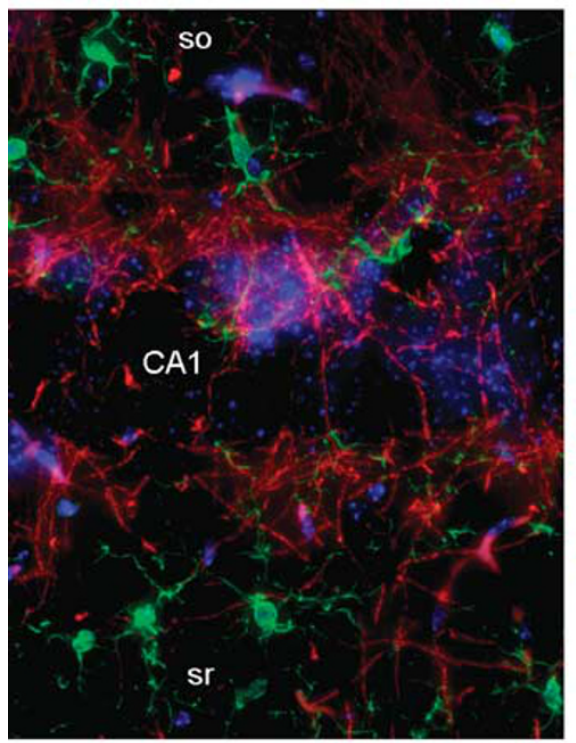

b

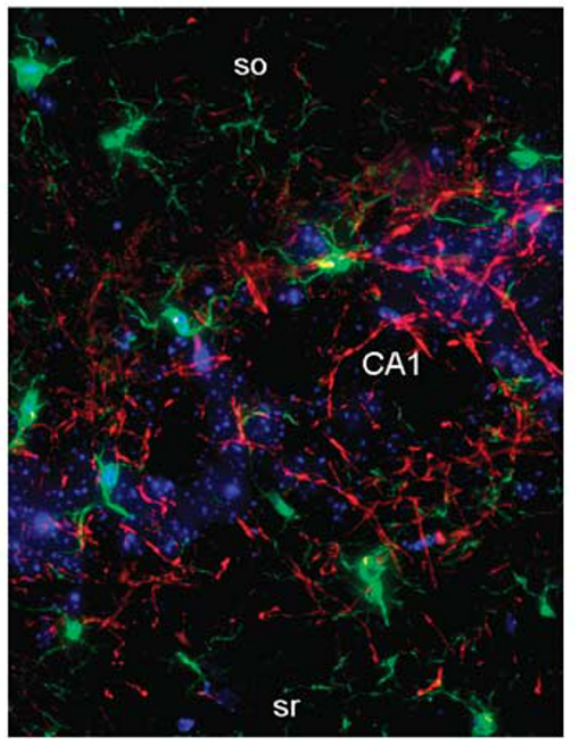

C

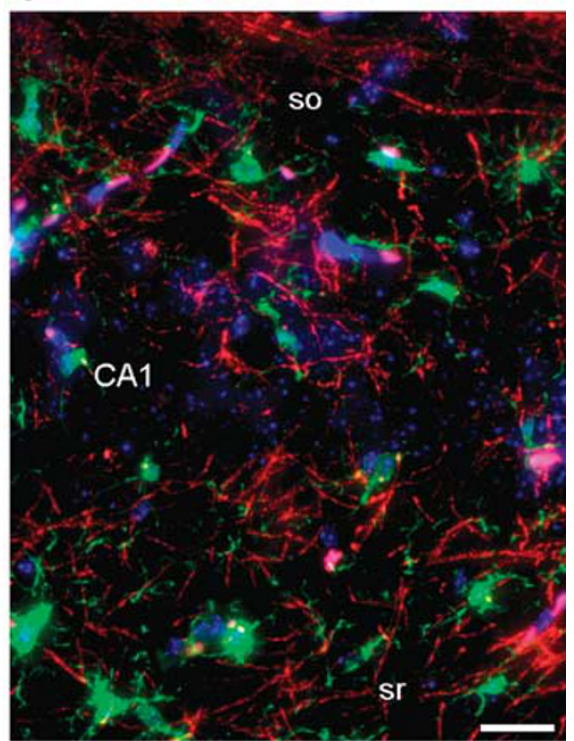

d
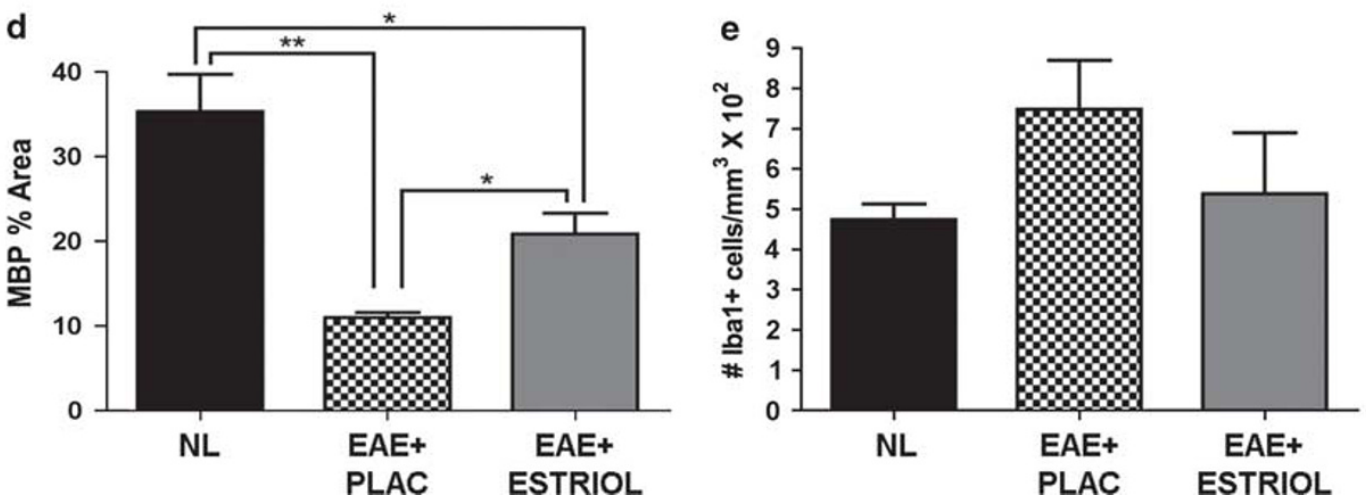

Figure 5 Decreased myelin staining and increased microglial activation occur within the hippocampus during EAE, and estriol treatment preserves myelin staining. (a-c) Pseudo-colored confocal fluorescent images depict myelin basic protein immunoreactivity (MBP, Cy5-red) and cells of microglial/macrophage lineage stained with Iba1 (Cy3-green) and DAPI (blue) in the CA1 region within healthy (a), placebo-treated EAE (b) and estriol-treated EAE (c) mice at $\times 40$ magnification. (d) One-way ANOVA revealed significant differences in myelin staining across conditions, $P=0.003, n=5$ mice per group, and Bonferroni post-hoc tests indicated that EAE caused a significant reduction in myelin staining within the CA1 region of the hippocampus in placebo-treated mice (EAE + PLAC, ${ }^{* * P}<0.01, n=5$ mice) compared with myelin staining in hippocampus of healthy control mice (NL, $n=5$ mice). Estriol-treated EAE mice (EAE + ESTRIOL, $n=5$ mice) had myelin levels significantly increased compared with placebo-treated EAE mice $\left({ }^{*} P<0.05\right)$, but were still significantly different from myelin staining levels in healthy controls. Analysis was conducted on $\times 10$ magnification images where MBP percent area represents total MBP immunoreactivity as a percent of total delineated CA1 area imaged. Iba $1+$ cells with morphology characteristic of reactive microglia were relatively low in healthy controls (a), and increased in placebo-treated EAE mice (b). However, the number of lba1 + cells was not significantly different in estrioltreated EAE mice (c). The number of Iba $1+$ cells per $\mathrm{mm}^{3}$ were counted and presented as whole numbers (e). Data are representative of two experiments. Scale bar, $10 \mu \mathrm{m}$.

\section{Neuropathological Outcome Measures Are Significantly Correlated with Changes in Electrophysiological Outcome Measures}

Because we found that excitatory synaptic transmission was altered in hippocampal slices from placebo-treated EAE mice and also found neuropathology in tissue from those same animals, we next used cross-modality correlations to determine if functional and pathological outcomes were related during disease state. Specifically, we investigated if changes in PSD-95 and NRXII staining levels correlated with changes in excitatory synaptic transmission. Interestingly, PSD-95 percent area in disease states was significantly correlated to
fEPSP responses during disease states (Figure 7a). Furthermore, the number of immunoreactive NRXII puncta was also significantly correlated to fEPSP (Figure $7 \mathrm{~b}$ ) during disease states. Thus, these cross-modality correlations together demonstrate that excitatory synaptic transmission during EAE coincides with levels of PSD-95 and NRXII $\beta$ in the hippocampus during EAE.

\section{DISCUSSION}

The results of the present study show, for the first time, that excitatory synaptic transmission and PPF are significantly impaired in the hippocampus during EAE and that estriol 


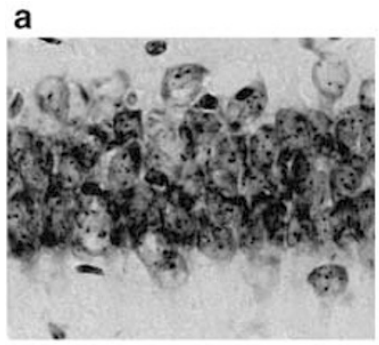

b

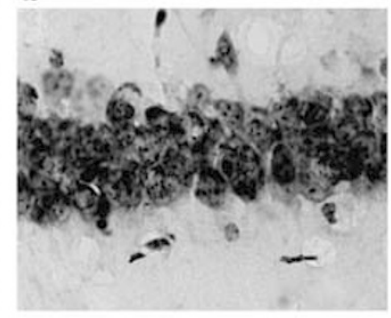

c

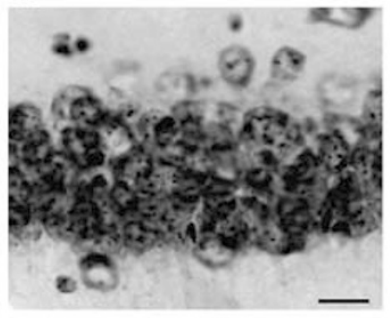

d

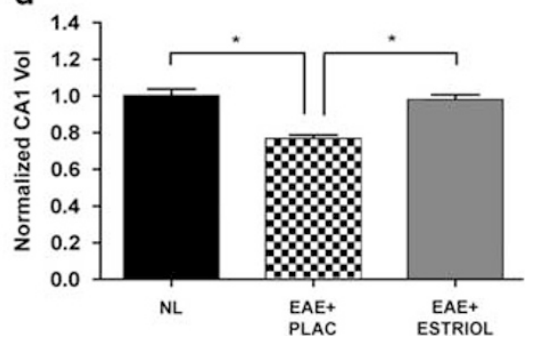

Figure 6 Hippocampal CA1 atrophy occurs during EAE and estriol treatment prevents this. (a-c) Nissl-stained hippocampal sections representative of normal (NL; a), placebo-treated EAE (EAE + PLAC; b) and estriol-treated EAE (EAE + ESTRIOL; $\mathbf{c})$ mice depict the CA1 pyramidal layer at $\times 40$ magnification. Compared with healthy controls, placebo-treated EAE mice had significantly reduced CA1 pyramidal layer volume, quantified in panel d. With estriol treatment during EAE, the CA1 pyramidal volume was preserved to levels similar to those in controls. One-way ANOVA and Bonferroni post-hoc analysis revealed significant difference between three groups $\left({ }^{\star} P=0.05, n=5\right.$ mice per group), scale bar, $10 \mu \mathrm{m}$. This finding has been confirmed by repeated experiments.
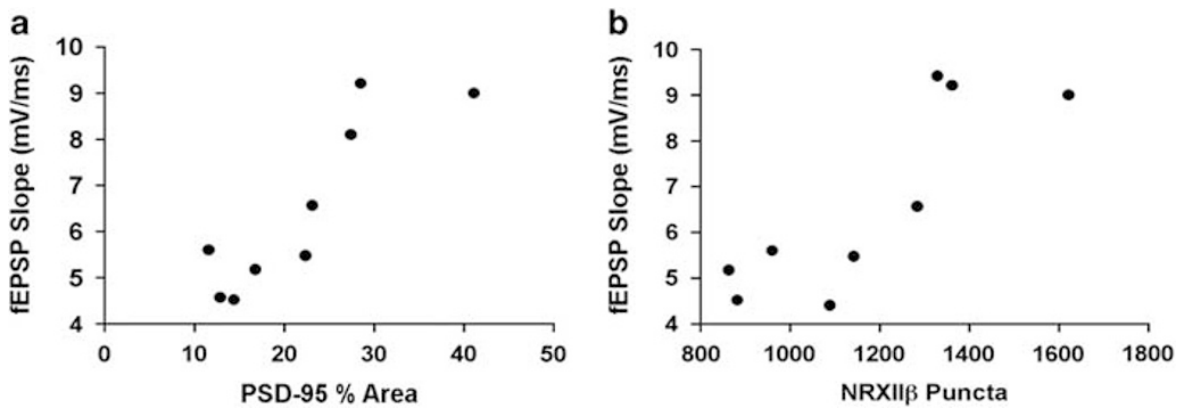

Figure 7 Decreased pre- and post-synaptic protein levels are significantly correlated with electrophysiological changes during EAE. Cross-modality correlations were conducted to determine potential neuropathological substrates underlying changes in excitatory synaptic transmission and paired-pulse facilitation during EAE. (a) PSD-95 percent area was significantly correlated to maximal fEPSP slope throughout the experiment, where the correlation coefficient rho $=0.8767, P=0.019, n=9$ mice total. (b) The average number of presynaptic NRXII $\beta$ puncta was also significantly correlated to postsynaptic responses (fEPSP) where rho $=0.8396, P=0.0046, n=9$ mice total.

treatment is capable of preventing this. Furthermore, we demonstrate that these functional deficits occur within tissue that has neuropathological changes in expression of synaptic proteins known to play an important role in excitatory transmission in the hippocampus (PSD-95, NRXII $\beta$ and NLG1). Interestingly, estriol treatment prevented these changes in synaptic protein expression.

Our data show a reduction of both pre- and post-synaptic electrophysiological responses in hippocampal slices of mice with EAE. Presynaptic facilitation is inversely related to synaptic vesicle release probability at presynaptic terminals. ${ }^{50,51}$ As we found elevated PPF during disease, this suggested that EAE decreased the probability of presynaptic vesicle release. Presynaptic terminal structure and protein composition can directly mediate changes in the probability of synaptic vesicle release. ${ }^{52,53}$ Thus, it is plausible that EAE leads to detrimental changes in presynaptic terminal structure and protein composition. Postsynaptic excitatory responses were also reduced in this study, and thus EAE may cause simultaneous alterations to pre- and post-synaptic terminals that lead to impaired synaptic transmission.

EAE induced a reduction in protein levels of trans-synaptic binding partners. PSD-95, NRXII $\beta$ and NLGI are distinct, interacting scaffolding proteins known to have important roles in excitatory synaptic transmission. ${ }^{54}$ PSD95 levels were significantly decreased in the hippocampus of mice with EAE. PSD-95 is abundantly expressed in healthy excitatory synapses and has been shown to recruit diverse proteins to sites of synaptic adhesion ${ }^{55}$ and promote transsynaptic signaling. ${ }^{56}$ PSD-95 also has extensive interactions with NLG1 (a postsynaptic cell adhesion protein) at excitatory synapses ${ }^{57}$ and with NRXII $\beta$ (a presynaptic cell adhesion protein) to mediate presynaptic terminal assembly. ${ }^{58}$ Indeed, neurexin/neuroligin trans-synaptic binding is necessary for proper synapse formation, stability, maturation and function. ${ }^{59}$ Both NRXII $\beta$ and NLG1 protein levels were reduced in the hippocampus during EAE and, moreover, cross-modality correlations demonstrated that the reductions in these protein levels were correlated with reductions in basal synaptic transmission and PPF. PSD-95 and neuroligin can directly modulate the release probability of neurotransmitter vesicles in a retrograde fashion via direct binding with neurexin. ${ }^{47}$ Thus, the changes in these synaptic protein levels during EAE may affect probability of vesicle release, and result in impairment of hippocampal function. 
Estrogens are known to have extensive effects on the hippocampus in healthy and disease states, making them likely candidates for neuroprotective treatments. Estrogens promote synaptogenesis ${ }^{60}$ and can enhance synaptic transmission in healthy states ${ }^{61}$ by facilitating presynaptic and postsynaptic plasticity. ${ }^{62,63}$ In addition, estrogens can significantly increase expression of synaptic proteins required for functional synaptic transmission, including PSD-95 ${ }^{64}$ and neuroligin. ${ }^{65}$ Besides providing beneficial support to synaptic integrity and function in healthy conditions, estrogens have also been shown to induce other neuroprotective mechanisms in other diseases. ${ }^{66}$ In animal models of Alzheimer's disease, estrogen treatment reduced $\beta$-amyloid protein accumulation, ${ }^{67}$ promoted neuron viability ${ }^{68}$ and enhanced NMDA-mediated synaptic transmission and long-term plasticity. ${ }^{63}$ In EAE, estrogen treatment has been shown to reduce clinical disease severity $^{18,19}$ through both neuroprotective and anti-inflammatory actions. ${ }^{20,21,42}$ As EAE principally affects ambulation, the majority of these studies have been limited to spinal cord pathology. One recent publication has examined estrogen-mediated neuroprotection in the corpus callosum ${ }^{12}$ and found that estrogen treatment prevented callosal axon loss and preserved axonal conductance across the corpus callosum. Our study is the first to report that estriol, a pregnancy estrogen, is capable of preventing EAE-mediated impairment hippocampal function and neuropathology. Important evidence from human clinical trials in MS suggests that estriol treatment may be beneficial in clinical application ${ }^{15,17,69}$ and further investigation in clinical trials is underway (http:// clinicaltrials.gov/ct2/show/NCT00451204). This report suggests that estriol treatment should be further investigated for its potential role in preventing hippocampal dysfunction and neuropathology in MS.

It remains to be determined where and how estriol might act to prevent the pathological changes that occur in the hippocampus during EAE. To understand the cellular and molecular mechanism of estrogen-mediated protection in the hippocampus during EAE, treatment with estrogen-receptorspecific ligands must be investigated. Estrogen-mediated protection, like other estrogenic actions, is mediated primarily through estrogen receptors, $\mathrm{ER} \alpha$ and $\mathrm{ER} \beta$, although other non-genomic mechanisms have been identified. $\operatorname{ER} \alpha$ and $\operatorname{ER} \beta$ have distinct tissue distributions, ${ }^{70}$ which may cause some tissue selectivity using selective estrogen receptor modifiers (SERMs). The two receptors act synergistically in some tissues, whereas they act antagonistically in others. These tissue-specific differences are thought to be because of tissue-specific differences in transcription factor activation upon ER-ligand binding. In a previous publication, our lab has shown that $\mathrm{ER} \alpha$ ligand (PPT) treatment ameliorated clinical disease score throughout disease, whereas treatment with an $\operatorname{ER} \beta$ ligand (DPN) had no effect in early EAE, but promoted recovery during the late phase of disease. ${ }^{21}$ In addition, EAE mice treated with ER $\alpha$ ligand had significantly reduced CNS inflammation, whereas EAE mice treated with ER $\beta$ ligand did not. Interestingly, treatment with either the $\mathrm{ER} \alpha$ or the $\mathrm{ER} \beta$ ligand was neuroprotective, as evidenced by axonal sparing within the white matter of the spinal cord. By using ER-specific ligands, the anti-inflammatory effects were dissociated from the neuroprotective effects of estrogen treatment during $\mathrm{EAE}$, as only ER $\alpha$ ligand treatment significantly reduced inflammation in the spinal cord. These data suggested that the neuroprotective effects of estrogen treatment are not necessarily dependent on anti-inflammatory properties, but rather may be mediated by direct effects of estrogen binding to estrogen receptors on cells within the central nervous system. Estrogen receptors have been detected on several CNS cells, including neurons, ${ }^{71,72}$ astrocytes, ${ }^{73}$ oligodendrocytes and microglia. ${ }^{74}$ Recently, it was shown that when ER $\alpha$ was selectively removed from astrocytes, but not neurons, the protection from spinal cord pathology in ER $\alpha$ ligand-treated EAE mice was lost. Both $\operatorname{ER} \alpha$ and $\operatorname{ER} \beta$ are expressed in the hippocampus. ${ }^{75}$ Future studies using CNS cell-specific ER knockouts are needed to determine whether the astrocyte or other CNS cell is the target of estriol-mediated protection in the hippocampus during EAE.

In conclusion, this is the first report demonstrating that EAE leads to impaired synaptic transmission and corresponding changes in synaptic protein levels within the hippocampus. Moreover, our data argue strongly that estriol treatment is neuroprotective in the hippocampus by preventing both dysfunction and neuropathology. Although there are always limitations to the conclusions that one can draw from animal models of disease, this report provides a foundation for further studies that focus on hippocampal synaptic proteins as they relate to hippocampal-dependent cognitive impairment in MS.

\section{ACKNOWLEDGEMENTS}

This work was supported by the National Institutes of Health (Grant K24NS052117) and from the National Multiple Sclerosis Society (Grants RG4033 and RG4364 to RRV), as well as funding from the Skirball Foundation, the Conrad Hilton Foundation and the Sherak Family Foundation. MOZ was supported in part by the UCLA Laboratory of Neuroendocrinology (LNE), funded by the National Institutes of Health (Grant T32 HD07228-26), and in part by the National Science Foundation GK-12 UCLA SEE-LA program (Grant DGE-074241). This work was also supported by the National Institutes of Mental Health (Grant MH609197 to TJO).

\section{DISCLOSURE/CONFLICT OF INTEREST}

The authors declare no conflict of interest.

1. Chiaravalloti ND, DeLuca J. Cognitive impairment in multiple sclerosis. Lancet Neurol 2008;7:1139-1151.

2. Benedict RHB, Cookfair D, Gavett $R$, et al. Validity of the minimal assessment of cognitive function in multiple sclerosis (MCFIMS). J Int Neuropsychol Soc 2006;12:549-558.

3. Thornton $A E$, Raz N. Memory impairment in multiple sclerosis. Neuropsychology 1997;11:357-366.

4. Benedict RHB, Ramasamy D, Munschauer F, et al. Memory impairment in multiple sclerosis: correlation with deep grey matter and mesial temporal atrophy. J Neurol Neurosurg Psychiatry 2009;80:201-206.

5. Roosendaal SD, Moraal $\mathrm{B}$, Vrenken $\mathrm{H}$, et al. In vivo imaging of hippocampal lesions in multiple sclerosis. J Magn Reson Imaging 2008; 27:726-731. 
6. Anderson VM, Fisniku LK, Khaleeli A, et al. Hippocampal atrophy in relapsing-remitting and primary progressive MS: a comparative study. Mult Scler 2010;16:1083-1090.

7. Sicotte NL, Kern KC, Giesser BS, et al. Regional hippocampal atrophy in multiple sclerosis. Brain 2009;132:3072-3086.

8. Geurts JJ, Bo L, Roosendaal SD, et al. Extensive hippocampa demyelination in multiple sclerosis. J Neuropathol Exp Neurol 2007;66: 819-827.

9. Papadopoulos D, Dukes S, Patel R, et al. Substantial archaeocortical atrophy and neuronal loss in multiple sclerosis. Brain Pathol 2009;19: 238-253.

10. Dutta R, Chang A, Doud MK, et al. Demyelination causes synaptic alterations in hippocampi from Multiple Sclerosis patients. Ann Neurol 2011;69:445-454.

11. Rasmussen $S$, Wang $Y$, Kivisakk $P$, et al. Persistent activation of microglia is associated with neuronal dysfunction of callosal projecting pathways and multiple sclerosis-like lesions in relapsing remitting experimental autoimmune encephalomyelitis. Brain 2007;130:2816-2829.

12. Crawford DK, Mangiardi M, Song B, et al. Oestrogen receptor beta ligand: a novel treatment to enhance endogenous functional remyelination. Brain 2010;133:2999-3016.

13. MacKenzie-Graham A, Tiwari-Woodruff SK, Sharma G, et al. Purkinje cell loss in experimental autoimmune encephalomyelitis. Neuroimage 2009;48:637-651.

14. Centonze D, Muzio L, Rossi S, et al. Inflammation triggers synaptic alteration and degeneration in experimental autoimmune encephalomyelitis. J Neurosci 2009;29:3442-3452.

15. Gold SM, Voskuhl RR. Estrogen and testosterone therapies in multiple sclerosis. Prog Brain Res 2009;175:239-251.

16. Confavreux C, Hutchinson M, Hours MM, et al. Rate of pregnancyrelated relapse in multiple sclerosis. Pregnancy in Multiple Sclerosis Group. N Engl J Med 1998;339:285-291.

17. Sicotte NL, Liva SM, Kluth R, et al. Treatment of multiple sclerosis with the pregnancy hormone estriol. Ann Neurol 2002;52:421-428.

18. Kim S, Liva SM, Dalal MA, et al. Estriol ameliorates autoimmune demyelinating disease: implications for multiple sclerosis. Neurology 1999:52:1230-1238.

19. Bebo Jr BF, Fyfe-Johnson A, Adlard K, et al. Low-dose estrogen therapy ameliorates experimental autoimmune encephalomyelitis in two difference inbred mouse strains. J Immunol 2001;166:2080-2089.

20. Morales LBJ, Loo KK, Liu HB, et al. Treatment with an estrogen receptor \{alpha\} ligand is neuroprotective in experimental autoimmune encephalomyelitis. J Neurosci 2006;26:6823-6833.

21. Tiwari-Woodruff $\mathrm{S}$, Morales LBJ, Lee $\mathrm{R}$, et al. Differential neuroprotective and anti-inflammatory effects of estrogen receptor (ER) $\alpha$ and ER $\beta$ ligand treatment. Proc Natl Acad Sci USA 2007;104: 14813-14818.

22. Vetego $\mathrm{E}$, Benedusi $\mathrm{V}$, Maggi $\mathrm{A}$. Estrogen anti-inflammatory activity in the brain: a therapeutic opportunity for menopause and neurodegenerative diseases. Front Neuroendocrinol 2008;29:507-519.

23. Stein DG, Hoffman SW. Estrogen and progesterone as neuroprotective agents in the treatment of acute brain injuries. Pediatr Rehabil 2003 6:13-22.

24. Wise PM, Dubal DB, Rau SW, et al. Are estrogens protective or risk factors in brain injury and neurodegeneration? Reevaluation after the Women's Health Initiative. Endocr Rev 2005;26:308-312.

25. Behl C, Skutella T, Lezoualch F, et al. Neuroprotection against oxidative stress by estrogens: structure-activity relationship. Mol Pharmacol 1997;51:535-541.

26. Murphy DD, Cole NB, Greenberger V, et al. Estradiol increases dendritic spine density by reducing GABA neurotransmission in hippocampal neurons. J Neurosci 1998;18:2550-2559.

27. Jover $\mathrm{T}$, Tanaka $\mathrm{H}$, Calderone $\mathrm{A}$, et al. Estrogen protects against global ischemia-induced neuronal death and prevents activation of apoptotic signaling cascades in the hippocampal CA1. J Neurosci 2002;22: 2115-2124.

28. Kretz O, Fester L, Wehrenberg U, et al. Hippocampal synapses depend on hippocampal estrogen synthesis. J Neurosci 2004;24:5913-5921.

29. Toran-Allerand CD. Novel sites and mechanism of oestrogen action in the brain. Novartis Found Symp 2000;230:56-73.

30. Kramar EA, Chen LY, Brandon NJ, et al. Cytoskeletal changes underlie estrogen's acute effects on synaptic transmission and plasticity. J Neurosci 2009;29:12982-12993.
31. Yun $\mathrm{SH}$, Park KA, Kwon $\mathrm{S}$, et al. Estradiol enhances long-term potentiation in hippocampal slices from aged apoE4-TR mice. Hippocampus 2007;17:1153-1157.

32. Woolley CS. Effects of estrogen in the CNS. Curr Opin Neurobiol 1999;9:349-354.

33. Woolley CS. Acute effects of estrogen on neuronal physiology. Annu Rev Pharmacol Toxicol 2007;47:657-680.

34. Ziehn MO, Avedisian AA, Tiwari-Woodruff S, et al. Hippocampal CA1 atrophy and synaptic loss during experimental autoimmune encephalomyelitis, EAE. Lab Invest 2010;90:774-786.

35. Pettinelli $C$, McFarlin DE. Adoptive transfer of experimental allergic encephalomyelitis in SJL/J mice after in vitro activation of lymph node cells by myelin basic protein: requirement for Lyt 1+ 2- T lymphocytes. J Immunol 1981;127:1420-1423.

36. Cuthbert PC, Stanford LA, Coba MP, et al. Synapse-associated protein 102/dlgh3 couples the NMDA receptor to specific plasticity pathways and learning strategies. J Neurosci 2007;27:2673-2682.

37. Komiyama NH, Watabe AM, Carlisle HJ, et al. SynGAP regulates ERK/ MAPK signaling, synaptic plasticity, and learning in the complex with postsynaptic density 95 and NMDA receptor. J Neurosci 2002;22: 9721-9732.

38. Wiltgen BJ, Royle GA, Gray EE, et al. A role for calcium-permeable AMPA receptors in synaptic plasticity and learning. PLoS One 2010; 5:pii e12818.

39. Paxinos G, Franklin KBJ. The Mouse Brain in Stereological Coordinates, 2nd edn Academic Press: San Diego, CA, 2001.

40. Gundersen HJ, Jensen EB. The efficiency of systematic sampling in stereology and its prediction. J Microsc 1987;147:229-263.

41. Cruz-Orive LM. Precision of Cavalieri sections and slices with local errors. J Microsc 1999;193:182-198.

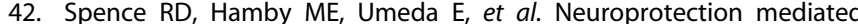
through estrogen receptor-alpha in astrocytes. Proc Natl Acad Sci USA 2011;108:8867-8872.

43. Arezzo JC, Litwak MS, Zotova EG. Correlation and dissociation of electrophysiology and histopathology in the assessment of toxic neuropathy. Toxicol Pathol 2011;39:46-51.

44. Aubert S, Wendling F, Regis J, et al. Local and remote epileptogenicity in focal cortical dysplasias and neurodevelopmental tumours. Brain 2009;132:3072-3086.

45. Lehmann HC, Zhang J, Mori S, et al. Diffusion tensor imaging to assess axonal regeneration in peripheral nerves. Exp Neurol 2010;223: 238-244.

46. Palaszynski KM, Liu H, Loo KK, et al. Estriol treatment ameliorates disease in males with experimental autoimmune encephalomyelitis: implications for multiple sclerosis. J Neuroimmunol 2004;149:84-89.

47. Futai K, Kim MJ, Hashikawa $\mathrm{T}$, et al. Retrograde modulation of presynaptic release probability through signaling mediated by PSD-95neuroligin. Nat Neurosci 2007;10:186-195.

48. Kuzumaki N, Ikegami D, Imai S, et al. Enhanced IL-1beta production in response to the activation of hippocampal glial cells impairs neurogenesis in aged mice. Synapse 2010;64:721-728.

49. Voskuhl RR, Peterson RS, Song B, et al. Reactive astrocytes form scarlike perivascular barriers to leukocytes during adaptive immune inflammation of the CNS. J Neurosci 2009;29:11511-11522.

50. Debanne D, Guerineau NC, Gahwiler BH, et al. Paired-pulse facilitation and depression at unitary synapses in rat hippocampus: quantal fluctuation affects subsequent release. J Physiol 1996;15(Pt 1):163-176.

51. Dobrunz LE, Stevens CF. Heterogeneity of release probability, facilitation and depletion at central synapses. Neuron 1997;18: 995-1008.

52. Zucker RS, Regehr WG. Short-term synaptic plasticity. Annu Rev Physiol 2002;64:355-405.

53. Conti $\mathrm{R}$, Lisman J. The high variance of AMPA receptor- and NMDA receptor-mediated responses at single hippocampal synapses: evidence for multi-quantal release. Proc Natl Acad Sci 2003;100: 4885-4890.

54. Dean C, Dresbach T. Neuroligins and neurexins: linking cell adhesion, synapse formation and cognitive function. Trends Neurosci 2006;29: 21-29.

55. Kim E, Sheng M. PDZ domain proteins of synapses. Nat Rev Neurosci 2004;5:771-781.

56. Han K, Kim E. Synaptic adhesion molecules and PSD-95. Prog Neurobiol 2008;84:263-283. 
57. Nguyen T, Sudhof TC. Binding properties of neuroligin 1 and neurexin 1 beta reveal function as heterophilic cell adhesion molecules. J Biol Chem 1997:272:26032-26039.

58. Dean C, Scholl FG, Choih J, et al. Neurexin mediates the assembly of presynaptic terminals. Nat Neusci 2003;6:708-716.

59. Levinson JN, Chery N, Huang K, et al. Neuroligins mediate excitatory and inhibitory synapse formation: involvement of PSD-95 and neurexin-1beta in neuroligin-induced synaptic specificity. J Biol Chem 2005;280:17312-17319.

60. Jelks $\mathrm{KB}$, Wylie R, Floyd $\mathrm{CL}$, et al. Estradiol targets synaptic proteins to induce glutamatergic synapse formation in cultured hippocampal neurons: critical role of estrogen receptor-alpha. J Neurosci 2007;27: 6903-6913.

61. Teyler TJ, Vardaris RM, Lewis D, et al. Gonadal steroids: effects on excitability of hippocampal pyramidal cells. Science 1980;209: 1017-1018.

62. Smejkalova T, Woolley CS. Estradiol acutely potentiates hippocampal excitatory synaptic transmission through a presynaptic mechanism. J Neurosci 2010;30:16137-16148.

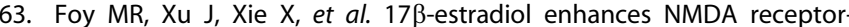
mediated EPSPs and long-term potentiation. J Neurophysiol 1999; 81:925-929.

64. Li C, Brake WG, Romeo RD, et al. Estrogen alters hippocampal dendritic spine shape and enhances synaptic protein immunoreactivity and spatial memory in female mice. Proc Natl Acad Sci USA 2004;101: 2185-2190.

65. Kang HS, Lee CK, Kim JR, et al Gene expression analysis of the prooestrous-stage rat uterus reveals neuroligin 2 as a novel steroidregulated gene. Reprod Fertil Dev 2004;16:763-772.
66. Garcia-Segura LM, Azcoitia I, DonCarlos LL. Neuroprotection by estradiol. Prog Neurobiol 2001;63:29-60.

67. Carroll JC, Rosario ER, Chang L, et al. Progesterone and estrogen regulate Alzheimer-like neuropathology in female 3xTg-AD mice. J Neurosci 2007;27:13357-13365.

68. Pike CJ, Carroll JC, Rosario ER, et al. Protective actions of sex steroid hormones in Alzheimer's disease. Front Neuroendocrinol 2009;30: 239-258.

69. Voskuhl RR. 'Estrogens in the Treatment of MS' Multiple Sclerosis Therapeutics, 3rd edn Informa Healthcare: UK, 2007, pp 645-657.

70. Enmark E, Gustafsson JA. Oestrogen receptors - an overview. J Intern Med 1999;246:133-138.

71. Mehra RD, Sharma K, Nyakas C, et al. Estrogen receptor alpha and beta immunoreactive neurons in normal adult and aged female rat hippocampus: a qualitative and quantitative study. Brain Res 2005; 1056:22-35.

72. Pfaff DW, Ribiero AC. Theoretical consequences of fluctuating versus constant liganding of oestrogen receptor-alpha in neurons. J Neuroendocrinol 2010;22:486-491.

73. Azcoitia I, Santos-Galindo M, Arevalo MA, et al. Role of astroglia in the neuroplastic and neuroprotective actions of estradiol. Eur J Neurosci 2010;32:1995-2002.

74. Arevalo MA, Santos-Galindo M, Bellini MJ, et al. Actions of estrogens on glial cells: implications for neuroprotection. Biochim Biophys Acta 2010;1800:1106-1112.

75. Mitterling KL, Spencer JL, Dziedzic N, et al. Cellular and subcellular localization of estrogen and progestin receptor immunoreactivities in the mouse hippocampus. J Comp Neurol 2010;518: 2729-2743. 